\title{
Cross-Disciplinary Research in Biology Education: Challenges and Opportunities
}

\section{Ido Davidesco ${ }^{\text {t* }}$ and Kimberly D. Tanner*}

'Department of Educational Psychology, Neag School of Education, University of Connecticut, Storrs, CT 06269-3064; „Department of Biology, San Francisco State University, San Francisco, CA 94132

I would love to collaborate with learning scientists, but I never meet them at biology education conferences.

Measuring students' brain activity? That sounds intriguing, but how would I get started?

What do you mean by "epistemological beliefs about science"? Oh, that's what we call "nature of science"!

Perhaps you have heard comments like the ones above or made them yourself. The challenges in initiating and conducting research across disciplinary boundaries are real. Researchers and practitioners from various disciplines attend different conferences, use different methodologies, and sometimes use different terms to refer to similar phenomena (see Peffer et al. in this issue). Further, individuals from across disciplines also do not usually publish their scholarly work in the same journals. This last point inspired this cross-disciplinary-themed issue of CBE-Life Sciences Education (LSE).

This special issue of the journal is a response to several recent calls to promote research across disciplinary boundaries in science, technology, engineering, and mathematics (STEM) education (e.g., Coley and Tanner, 2012; Peffer and Renken, 2016; McDaniel et al., 2017; Mestre et al., 2018). This special issue also reflects our professional backgrounds as co-guest editors. We initially met on another continent at the Latin American School for Education, Cognitive, and Neural Sciences, a conference designed to bring together, purposefully, researchers from a range of disciplinary backgrounds. This summer school in Argentina was designed to build new bridges between disciplines with the goal of developing effective evidence-based educational practices (Bruer, 2014). We immediately connected at the meeting, as we are both trained as neuroscientists and are conducting research on biology education, but with different methodological and theoretical approaches. When we returned to the United States, we brainstormed about how to draw attention to all the wonderful possibilities that could emerge from collaborations among biology education researchers and scholars doing research in other fields.

LSE has always strived to foster cross-disciplinary discussions and research. The journal's Current Insights feature, coauthored by editorial board members Sarah Eddy and Julia Gouvea, regularly highlights a broad range of new findings and articles from journals across different disciplines. In addition, previous special issues, such as Integrating Physics and Biology Education and Broadening Participation in STEM, have featured cross-disciplinary research. In considering submissions for this themed issue, we specifically invited research that: 1) drew from theoretical and empirical work in the social, educational, developmental, cognitive, or other forms of psychology, neuroscience, or the learning sciences; and 2) had implications for
CBE Life Sci Educ September 1, 2020 19:ed1 DOI:10.1187/cbe.20-07-0150

*Address correspondence to: Ido Davidesco (ido.davidesco(auconn.edu).

(c) 2020 I. Davidesco and K. Tanner. CBE-Life Sciences Education @ 2020 The American Society for Cell Biology. This article is distributed by The American Society for Cell Biology under license from the author(s). It is available to the public under an Attribution-NoncommercialShare Alike 3.0 Unported Creative Commons License (http://creativecommons.org/licenses/ by-nc-sa/3.0)

"ASCB $®$ " and "The American Society for Cell Biology $\AA^{\prime \prime}$ are registered trademarks of The American Society for Cell Biology. 
discipline-based biology education researchers and biology education practitioners.

The articles in this themed issue take important steps toward bridging different disciplines. Some of these studies extend existing research on biological representations and reasoning that have appeared in the journal in the past. Other studies use methodologies that are new to biology education research, such as electroencephalography, functional magnetic resonance imaging (fMRI), and learning analytics.

To give you a sense of the content of this special issue, we first attempted to classify the papers into disciplines, such as cognitive science, social psychology, and the learning sciences. However, we quickly realized that the lines between these disciplines are starting to blur. As such, we offer an overview that clusters articles into six categories that share commonalities of purpose, method, or topic area, some of which are approached from different disciplinary viewpoints, as well as additional explorations that go beyond this organization.

Two papers explore biological representations and reasoning, drawing on research in cognitive science: Menendez and colleagues study the design characteristics of life cycle diagrams; and Frey and colleagues explore students' concept-building tendencies and how they extend to classroom learning.

Two papers extend explorations of reasoning into the realm of knowledge transfer: Kaminske and colleagues review the cognitive psychology literature on transfer; and Slominski and colleagues investigate how experts reason about a crosscutting phenomenon across STEM disciplines.

Three papers probe the role of testing in learning: St. Clair and colleagues investigate how incentives modulate test-enhanced learning (i.e., the testing effect); Jensen and colleagues ask whether the testing effect generalizes to high-level learning; and Sana and colleagues explore how the efficacy of learning objectives can be enhanced with pretesting.

Two papers focus on psychosocial processes: Richardson and colleagues study how instructor mindset relates to teaching behaviors; Limeri and colleagues review research on psychosocial interventions and propose that it can be leveraged to increase instructors' motivation to participate in professional development.

Two papers incorporate brain measures in biology education research: Clark and colleagues use fMRI to study how modeling activities can support changes in students' neural representations; and Davidesco provides an overview of emerging research on brain-to-brain synchrony in STEM classrooms.
Finally, five papers explore other topics in teaching and learning using different disciplinary lenses: Peffer and colleagues use learning analytics to assess epistemological beliefs about science; Rhodes and colleagues build on cognitive load theory to investigate the value of case-based learning; Halmo and colleagues compare the efficacy of various pedagogies from educational psychology; Scott and colleagues discuss how design-based research can extend work in biology education; and we ourselves, in collaboration with Keller, introduce a framework for understanding attention in the classroom.

Taken together, the papers in this themed issue demonstrate that collaborations across biology education research and other disciplines, such as psychology, neuroscience, and the learning sciences, can benefit all disciplines involved. For example, such collaborations can allow psychology and neuroscience researchers to test their theories in the field, as biology education research is most often conducted in classrooms. These collaborations can also help biology education researchers incorporate new ideas and research methods from psychology and neuroscience.

We hope that this themed issue will help catalyze more cross-disciplinary research that extends the theoretical frameworks and methodologies from other disciplines into current questions in biology education. Additionally, we also hope readers will be inspired to engage in conversations with colleagues from other disciplines, learn new frameworks and methodologies, and continue to blur traditional boundaries and disciplinary lines.

\section{REFERENCES}

Bruer, J. T. (2014). The Latin American School on Education and the cognitive and neural sciences: Goals and challenges. Trends in Neuroscience and Education, 3(1), 1-3.

Coley, J. D., \& Tanner, K. D. (2012). Common origins of diverse misconceptions: Cognitive principles and the development of biology thinking. CBE-Life Sciences Education, 11(3), 209-215.

McDaniel, M. A., Mestre, J. P., Frey, R. F., Gouravajhala, R., Hilborn, R. C., Miyatsu, T., ... \& Slakey, L. L. (2017). Maximizing undergraduate STEM learning: Promoting research at the intersection of cognitive psychology and discipline-based education research. Retrieved July 15, 2020, from https://circle.wustl.edu/white-paper-maximizing-undergraduate -stem-learning

Mestre, J. P., Cheville, A., \& Herman, G. L. (2018). Promoting DBER-cognitive psychology collaborations in STEM education. Journal of Engineering Education, 107(1), 5-10.

Peffer, M., \& Renken, M. (2016). Practical strategies for collaboration across discipline-based education research and the learning sciences. CBELife Sciences Education, 15(4), es11. 